\title{
Evaluation of Biological Activity of Natural Composite Extracts
}

\author{
Hye-Jin Kwon, Professor, Dept. Chemical Engineering, Soongsil Univ., Dongjak-gu Seoul, Korea, 06978, \\ kwonhj0070@ssu.ac.kr \\ *Corresponding author
}

\begin{abstract}
To increase the value of using natural plants, we developed antioxidant and anti-agi ng cosmetic ingredients using Portulaca oleracea(PO) and Agrimonia pilosa Ledeb(APL), known a $s$ natural medicinal plants. Both PO and APL were extracted with ethanol as solvents and thec omposite extract was prepared in a 1:1 ratio of PO and APL extracts. The final samples wer e compared and evaluated based on three extracts, i.e., PO extract, composite extract, and APL extract. The antioxidant effects of these samples were evaluated by measuring DPPH, ABTS, a nd hydroxyl radical scavenging activity, whereas the anti-aging effects were evaluated via Coll agenase and Elastase inhibition rates. In evaluating the antioxidant activity of the APL extract was the highest, whereas that of the composite extract showed inhibitory activity similar to thatof the single extracts but still lower than that of individual extracts. Moreover, the anti-a ging effect of the composite extract demonstrated higher inhibitory activity than that of the si ngle

tract. The highest inhibition rate was shown in a 2:1 ratio of PO and APL composite. The re sults to date demonstrate that the composite extract of APL and PO has an efficacy to be used as a functional cosmetic material. With increased interest in anti-aging and well-being around $t$ he world, the development of natural materials for herbal medicine is expected to have a con siderable effect on the cosmetic industry in future.
\end{abstract}

Keywords: Portulaca oleracea; Agrimonia pilosa Ledeb; anti-aging; antioxidant; natural compo site extracts

Received: 05.12.2020 $\quad$ Accepted: 10.01.2021 $\quad$ Published: 06.02.2021

\section{INTRODUCTION}

The global cosmetics market is continuously growing. In particular, with the elderly population increasing, the "anti-aging cosmetics" are becoming a global trend. Moreover, the "composite function al cosmetics' with combined functions are attracting considerable attention along with "functional cosmetics" that are differentiated from ordinary cosmetics [1]. The popularity of natural cosmetics is primarily high in the Asia-Pacific region. In particular, natural cosmetic products containing plant extracts, organic ingredients, yeasts, and herbal medicine plants are being developed, as well as the demand for cosmetics that use natural ingredients is significantly increasing among composite functional product $s[2,3]$. In Korea, natural herbs have been used for their medicinal effects based on Donguibogam and several natural medicinal plants are already exploited as cosmetic ingredients [4]. Portulaca oleracea(P0) is a whole plant in Portulacaceae. Its pharmacological effects have been reported for antimicrobial, antioxidant, and anti-inflammatory activities; therefore, in oriental medicine, the plants are used in severa 1 inflammatory diseases. The major components of PO are coumarin, flavonoids, and glutamic acid; furthermore, its leaves and stalks were reported to contain high levels of essential fatty acids and nutritional components such as vitamin B1, B2, and malic acid [5]. However, Agrimonia pilosa Ledeb(APL) is used as a medicinal herb by drying the agrimony, the perennial herb of the Rosaceae family and the $t$ urning of the homologous plant. Generally, these plants are distributed in the roadsides and fields of the mountains in Korea. They have been used in folk medicine as hemostatic, anti-inflammatory, hypoglycemic, hematopoietic agents, and pain reliever. Moreover, it is being considered as having antiinflammatory, antibacterial, anti-cancer, and antiviral effects [6,7], other critical healthcare solutions [1834]. As part of basic research to explore antioxidant and anti-aging materials derived from natural products, we herein developed raw materials for antioxidant and anti-aging composite herbal cosmetic s using PO and APL; moreover, their biological activities were compared and evaluated. 


\section{Experimental setup \\ Sample extraction}

Portulaca oleracea (PO) and Agrimonia pilosa Ledeb (APL) used in this experiment were purchased at Kyungdong market in Seoul. Each sample (50 g) was extracted with $80 \%$ ethanol $(500 \mathrm{~mL}$ ) for $24 \mathrm{~h}$ at $60{ }^{\circ} \mathrm{C}$, centrifuged, filtered twice with filter paper, and concentrated under reduced pressure. The concentr ated sample was freeze-dried at $-80^{\circ} \mathrm{C}$ for $24 \mathrm{~h}$. The composite was prepared by mixing PO and APL in a 1:1 ratio and PO single extract, composite extract, and APL single extract were analyzed and compared f or final samples.

\section{Evaluation of antioxidant effects}

The antioxidant effects of samples were evaluated by DPPH, ABTS, and $\mathrm{OH}$ radical scavenging activities. In the DPPH experiment, $150 \mu \mathrm{L}$ of each sample was mixed with $100 \mu \mathrm{L}$ of $0.2 \mathrm{mM}$ DPPH solution in ethanol and reacted at $37{ }^{\circ} \mathrm{C}$ for $30 \mathrm{~min}$. After the reaction, absorbance was measured at $517 \mathrm{~nm}$. Note that distilled water was added to the control group of the sample, and ethanol was added to the control group of DPPH solution to obtain a correction value and the result was calculated. In the ABTS experiment, potassium persulphate solution $(2.6 \mathrm{mM})$ and ABTS $(7.4 \mathrm{mM})$ were mixed, and the mixture was stored in the dark for 1 day to form cations (ABTS'+). The absorbance was measured at $732 \mathrm{~nm}$. Next, the absorbance was diluted to its value being $<1.5$ and $5 \mu \mathrm{L}$ of each sample was mixed with diluted ABTS'+ solution $(150 \mu \mathrm{L})$ and reacting at room temperature for $10 \mathrm{~min}$. Moreover, absorbance was measured at $732 \mathrm{~nm}$. The distilled water was added to the control group of the sample; moreover, the result was obtained by measuring ABTS radical scavenging activity against the control group. For the $\mathrm{OH}$ radical

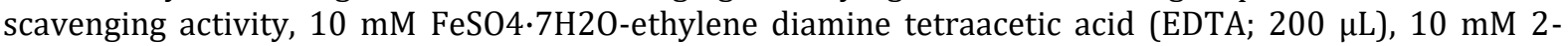
deoxyribose $(200 \mu \mathrm{L}), 10 \mathrm{mM}$ hydrogen peroxide $(200 \mu \mathrm{L})$, and $1400 \mu \mathrm{L}$ of the samples of each concentration were mixed and incubated at $37{ }^{\circ} \mathrm{C}$ for $4 \mathrm{~h}$. Note that $1 \mathrm{~mL}$ of $1.0 \%$ thiobarbituric acid and 1 $\mathrm{mL}$ of $2.8 \%$ trichloroacetic acid was added to the mixture and boiled for $20 \mathrm{~min}$. After cooling, the absorbance was measured at $490 \mathrm{~nm}$. All experimental results were statistically processed using SPSS 21.0.

\section{Evaluation of anti-aging effects}

Experiment on anti-aging was performed on collagenase and elastase inhibition rates. The sam ples were diluted to $1,10,100$, and $1,000 \mu \mathrm{g} / \mathrm{mL}$ and used. Both elastase and N-succinyl-(LAla) 3-p-nitroanilide were dissolved in $50 \mathrm{mM}$ Tris- $\mathrm{HCl}$ buffer $(\mathrm{pH} \mathrm{8.6)}$ as per the correspond ing concentrations. The $40-\mu \mathrm{L}$ sample was added with $40 \mu \mathrm{L}$ elastase of $2.5 \mathrm{U} / \mathrm{mL}$. Then, $80 \mu \mathrm{L}$ of $0.5 \mathrm{mg} / \mathrm{mL} \mathrm{N}$-succinyl-(L-Ala)3-p-nitroanilide was added and reacted at $37{ }^{\circ} \mathrm{C}$ for $30 \mathrm{~min}$ ' th e absorbance was measured at $445 \mathrm{~nm}$. Moreover, 4-phenylazobenzyloxycarbonyl-Pro-Leu-Gly-ProD-Arg was dissolved in $0.1 \mathrm{M}$ Tris- $\mathrm{HCl}$ buffer $(\mathrm{pH} 7.5)$ containing $4 \mathrm{mM} \mathrm{CaCl} 2$ and prepared. We added $250 \mu \mathrm{L}$ of $0.3 \mathrm{mg} / \mathrm{mL} 4$-phenylazobenzyloxycarbonyl-Pro-Leu-Gly-Pro-D-Arg to $100 \mu \mathrm{L}$ of the sample, followed by adding $150 \mu \mathrm{L}$ of collagenase $(0.2 \mathrm{mg} / \mathrm{mL})$. The resultant mixture was react ed at room temperature for $20 \mathrm{~min}$, and the absorbance was measured at $320 \mathrm{~nm}$ after adding $500 \mu \mathrm{L}$ of $6 \%$ citric acid and ethyl acetate $(1.5 \mathrm{~mL})$ to the reaction mixture. All results were sta tistically processed using SPSS 21.0.

\section{Result discussions}

\section{Antioxidant effects of the plant extracts}

Fig. 1, 2 shows the DPPH and ABTS radical scavenging activities of the single extract of PO and APL. At 1, 10,100 , and $1,000 \mu \mathrm{g} / \mathrm{mL}$, the DPPH radical scavenging activity of PO extract was $0.1 \% \pm 0.5 \%, 0.9 \% \pm$ $2.5 \%, 9.5 \% \pm 2.8 \%$, and $63.5 \% \pm 3.2 \%$, whereas that of APL extract was $2.6 \% \pm 0.4 \%, 6.5 \% \pm 0.4 \%, 41.4 \%$ $\pm 2.1 \%$, and $89.8 \% \pm 0.4 \%$, respectively. Both extracts seemed to be concentration-dependent and the APL extract showed more powerful antioxidant effects than the PO extract by concentration. In terms of the ABTS radical scavenging activity at $1,10,100$, and $1,000 \mu \mathrm{g} / \mathrm{mL}$, the PO extract was $0.24 \% \pm 0.3 \%$, $2.48 \% \pm 2.3 \%, 30.0 \% \pm 3.3 \%$, and $84.6 \% \pm 2.9 \%$, whereas that of the APL extract was $0.9 \% \pm 0.1 \%, 4.7 \%$ $\pm 0.5 \%, 33.4 \% \pm 0.4 \%$, and $94.7 \% \pm 0.2 \%$, respectively. Similar to the results of the DPPH radical scavenging activity, the ABTS radical scavenging activity of both extracts seemed to be concentrationdependent, whereas the APL extract showed higher antioxidant activity than the PO extract by concentra tion. Most natural products showed radical scavenging activity depending on concentrations. Thus, this $\mathrm{m}$ ethod is the most efficient tool to investigate whether natural products have antioxidant effects [8]. For applying natural products as cosmetic ingredients; however, it is important to determine optimal concentration. The result of the DPPH radical scavenging activity of the 1:1 mixture of PO and APL extracts was $0.05 \% \pm 1.4 \%, 0.3 \% \pm 3.2 \%, 2.4 \% \pm 2.2 \%$, and $10.5 \% \pm 0.3 \%$, whereas the ABTS radical scavenging activity was $0.05 \% \pm 1.4 \%, 1.1 \% \pm 0.4 \%, 3.6 \% \pm 0.2 \%$, and $24.4 \pm 0.3 \%$, indicating the anti-oxi 
dant activities of two single extracts were more effective than the composite extract. In general, the maximum effect of each compound is expected when two compounds are mixed [9, 10]. However, as shown in this study, its effect may be reduced to a certain extent. Table 1 summarizes the $\mathrm{OH}$ radical scavenging activities of the samples. It showed similar tendency to the results of two experiments $t$ hat occurred. The hydroxyl radical scavenging activity of the APL extract was the highest; however, the mixed extract was somewhat reduced.

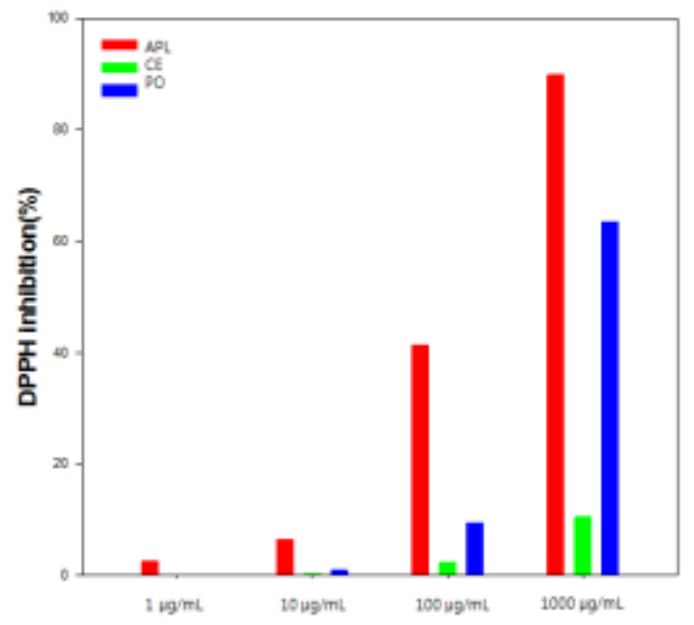

Figure 1. DPPH inhibition of the extract:

Portulaca oleracea (PO), Agrimonia pilosa Ledeb (APL), composite extract (CE)

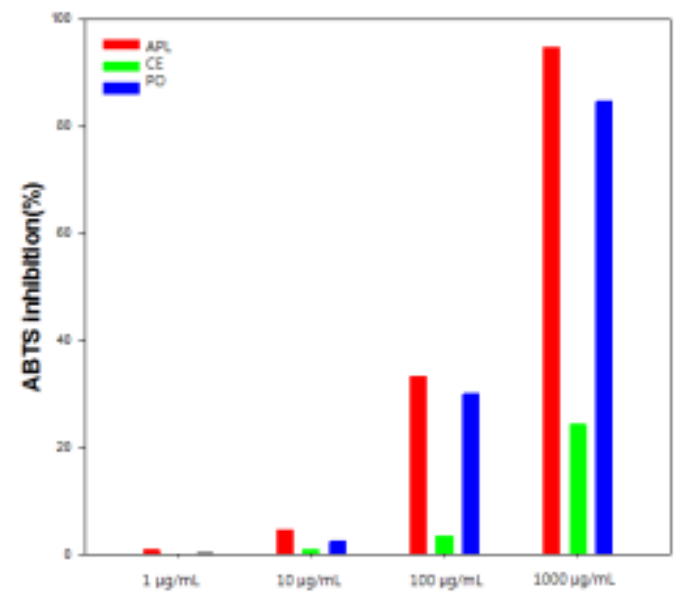

Figure 2. ABTS inhibition of the extract:

Portulaca oleracea (PO), Agrimonia pilosa Ledeb (APL), composite extract (CE)

Table 1. OH radical scavenging activities of the extracts

\begin{tabular}{|l|l|l|l|l|}
\hline concentration & $1 \mu \mathrm{g} / \mathrm{mL}$ & $10 \mu \mathrm{g} / \mathrm{mL}$ & $100 \mu \mathrm{g} / \mathrm{mL}$ & $1000 \mu \mathrm{g} / \mathrm{mL}$ \\
\hline APL & $1.04 \pm 0.61 \%$ & $3.92 \pm 0.05$ & $38.6 \pm 0.03$ & $90.59 \pm 0.29$ \\
\hline PO & $0.1 \pm 0.54 \%$ & $3.16 \pm 0.04$ & $37.7 \pm 0.03$ & $71.83 \pm 0.16$ \\
\hline CE & $0.6 \pm 0.26 \%$ & $1.5 \pm 0.47 \%$, & $21.6 \pm 7.2 \%$ & $40.1 \pm 0.06$ \\
\hline
\end{tabular}

\section{Anti-aging effects of the plant extracts}

Fig 3, 4 shows the anti-aging results of the three samples. In the APL extract, elastase inhibition rate was $6.4 \% \pm 0.9 \%, 19.4 \% \pm 1.0 \%, 38.0 \% \pm 0.5 \%, 60.4 \% \pm 1.2 \%$, and collagenase inhibition rate was $2.1 \% \pm$ 
$1.4 \%, 18.5 \% \pm 1.4 \%, 50.5 \% \pm 0.3 \%, 73.6 \% \pm 1.9 \%$ at $1,10,100$, and $1,000 \mu \mathrm{g} / \mathrm{mL}$, respectively. Both inhibition rates were concentration-dependent and higher than those of other recent studies, suggesting that the APL extract may be a promising candidate as a cosmeceutical ingredient with an anti-aging effe ct [11].

For the elastase inhibition activity experiment at $1,10,100$, and $1,000 \mu \mathrm{g} / \mathrm{mL}$, the P0 extract showed $9.1 \%$ $\pm 0.8 \%, 15.6 \% \pm 0.3 \%, 48.3 \% \pm 0.5 \%$, and $76.1 \% \pm 0.3 \%$ inhibition rates, whereas the composite extract (CE) showed $10.6 \% \pm 1.5 \%, 17.3 \% \pm 0.3 \%, 49.7 \% \pm 0.7 \%$, and $80.5 \% \pm 1.6 \%$ inhibition rates, respectively, thus being the most predominant inhibitory agent among the three samples. At the same concentrations, the collagenase inhibition rate of PO extract was $14.5 \% \pm 2.5 \%, 32.6 \% \pm 1.0 \%, 57.8 \% \pm 0.7 \%$, and $82.3 \%$ $\pm 1.5 \%$; however, that of the CE was $12.4 \% \pm 1.8 \%, 31.4 \% \pm 0.5 \%, 56.7 \% \pm 1.0 \%$, and $90.7 \% \pm 2.2 \%$. Moreover, similar to the elastase inhibition activity, CE demonstrated the highest inhibition rate among the three samples. Portulaca oleracea (PO) and Agrimonia pilosa Ledeb (APL) are used in various fields such as health supplements, medicinal herbal ingredients, and nutritional supplements. APL comprises pyrocatecholic tannin, ellagytannin, phenol, saponin, luteolin, and coumarin, all of which possessed antioxidant effects and saccharides such as glucosides. Moreover, PO contains antioxidant substances such as tannin, saponin, flavonoid, and beta-carotene and essential fatty acids such as omega-3 and alphalinolenic acid [12]. As reported earlier, these two compounds have excellent antioxidant effects because they contain various nutrients and biologically active substances [13].

Recently, to exhibit synergistic effects with each other, natural products are favored in diverse fields and rather than simply using one natural product, there is an increasing number of cases in which natural products are used as a composite extract [14]. The most important aspect in prescribing these extracts is the mixing ratio [15]. Thus, in these two anti-aging experiments, the anti-aging effects of the two extracts have a synergistic effect because the composite extract demonstrated the best efficiency.

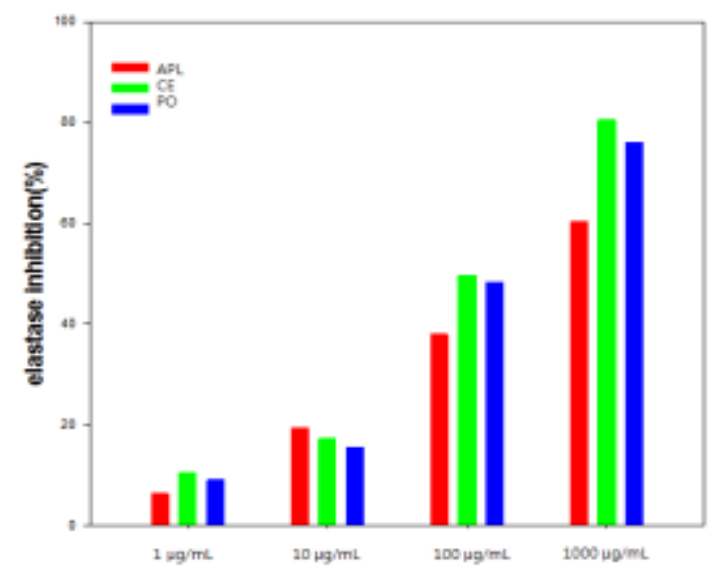

Figure 3. Elastase inhibition of the extract:

Portulaca oleracea (PO), Agrimonia pilosa Ledeb (APL), composite extract (CE)

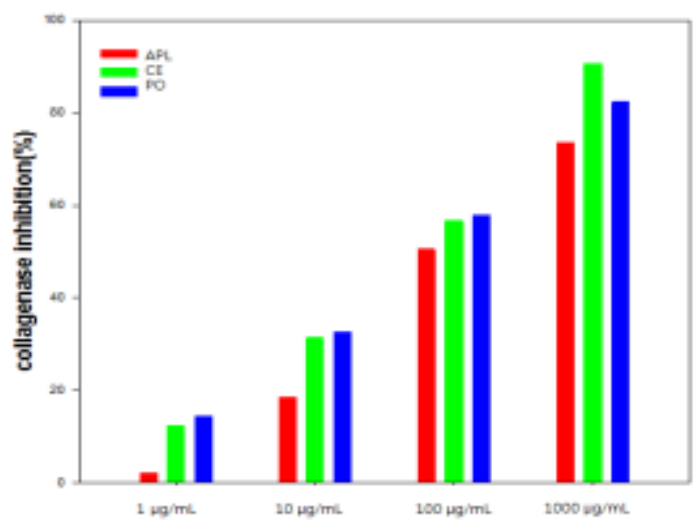

Figure 4. Collagenase inhibition of the extract:

Portulaca oleracea (PO), Agrimonia pilosa Ledeb (APL), composite extract (CE)

Figure 5, 6 shows the results of elastase and collagenase inhibitory activity at the mixing ratio of 2:1, 
1:1, and 2:1 (APL:PO). The mixture of 2:1 ratio showed the highest inhibition rates of $91.2 \% \pm 0.5 \%$ and $98.3 \% \pm 1.5 \%$ in elastase and collagenase inhibition experiments, respectively. Overall, the mixtures of 2:1 and 1:2 ratio showed improved anti-aging agents compared to the 1:1 mixture. Therefore, in the antiaging activity, we confirmed that the synergistic effect of the composite extract was evident, which did not appear in the antioxidant activity.

Coumarin is a phenolic secondary metabolite that is extensively distributed in plants and known for its anti-inflammatory, anti-cell aging, and anti-skin aging effects. Essential fatty acids such as omega 3 and alpha-linolenic acid are suggested to protect the skin and prevent skin aging [16]. As the skin ages, the skin loses its ability to produce lipid components, whereas the skin dries because of the lack of such lipid materials. Moreover, it tends to be damaged more easily because there are insufficient intercellular substances between the skin cells. Omega 3 can restore skin health and elasticity by strengthening skin barriers and preventing water loss in the skin [17]. Thus, for antioxidant and anti-aging cosmetics, the composite extracts of various antioxidant agents from APL and anti-aging agents from PO can be a promising functional ingredient.

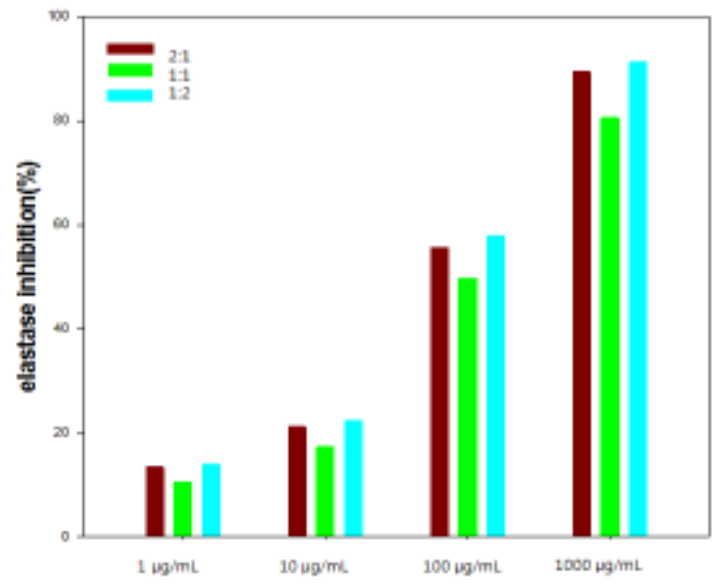

Figure 5. Elastase inhibition of the composite extract: $(2: 1,1: 1,1: 2)$

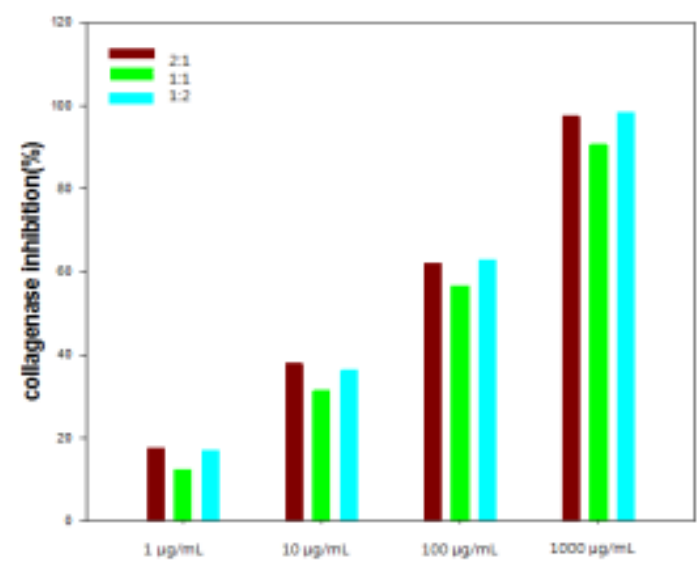

Figure 6. Collagenase inhibition of the composite extract: $(2: 1,1: 1,1: 2)$

\section{Conclusions}

This study aimed to increase the applicability of APL and PO composite extract as an ingredient for herbal cosmeceuticals and these conclusions were obtained. The antioxidant effect of the composite extract show ed inhibition rates similar to those of the single extract. However, for the anti-aging evaluation, the inhibitory effect of the mixed extract was higher than that of the single extract; nevertheless, the highest i nhibitory activity was observed in the 1:2 mixture of APL and PO. Accordingly, for cosmeceutical products, the composite extract was expected to be a suitable natural medicinal material. Recently, as people are increasingly interested in longevity without illness, interest and demand for functional ingredients are increasing. In particular, this trend is becoming more common because everybody is attempting to avoid using synthetic materials. Interests in natural medicinal herbs and their areas of use are continuously growing as both health supplements and raw materials for cosmetics. Most importantly, however, is the 
safety issue. These natural products have various advantages; however, they have disadvantages such as toxicity or adverse effects in certain individuals too. Therefore, continuous and systematic research on the stability of raw and herbal medicine from natural materials should be conducted.

\section{Acknowledgements}

This research was supported by 2018 Basic Science Research Program through the National Research Foundation of Korea funded by the Education, Science and Technology (No.2018008274).

\section{References}

[1] Nilforoushzadeh, M. A., Amirkhani, M. A., Zarrintaj, P., Moghaddam, A. S., Mehrabi, T., Alavi, S., Sisakht, M. M. (2018) Skin care and rejuvenation by cosmeceutical facial mask. J Cosmet Dermatol 17(5), 693702.

[2] Fan, L., Jia, Y., Cui, L., Li, X., He, C. (2017) Analysis of sensitive skin barrier function: basic indicators and sebum composition. Int J Cosmet Sci 40(2), 117-126.

[3] Kapoor, S., Saraf, S. (2010) Formulation and evaluation of moisturizer containing herbal extracts for the management of dry skin. Pharmacogn J 2(11), 409-417.

[4] Lee, B. K., Moon, S. J., Kim, H. S., Jung, Y. S. (2018) Anti-Atopic Activities of Mixture Herbal Aqua Extracts. Journal of the Korean Society of Food Science and Nutrition 47(10),1021-1028.

[5] Ahangarpour, A., Lamoochi, Z., Moghaddam, H. F., Mansouri S. M. T. (2016) Effects of Portulaca oleracea ethanolic extract on reproductive system of aging female mice. Int J Reprod BioMed 14(3), 205-212.

[6] Londonkar, R. L., Nayaka, H. B. (2013) Effect of ethanol extract of Portulaca oleracea L on ovulation and estrous cycle in female albino rats. J Pharm Res 6(4),431-436.

[7] Chondrogianni, N., Kapeta, S., Chinou, I., Vassilatou, K., Papassideri, I., Gonos, E. S. (2010) Anti-ageing and rejuvenating effects of quercetin. Exp Gerontol 45(10),763-771.

[8] Ahangarpour, A., Oroojan, A. A., Radan, M. (2014) Effect of aqueous and hydro-alcoholic extracts of lettuce (Lactuca sativa) seed on testosterone level and spermatogenesis in NMRI mice. Iran J Reprod Med 12(1),65-72.

[9] Litescu, S.C., Eremia, S. and Radu, G.L. (2010) Methods for the Determination of Antioxidant Capacity in Food and Raw Materials. Advances in Experimental Medicine and Biology 698, 241-249.

[10] Loginov, M., Boussetta, N., Lebovka, N. and Vorobiev, E. (2013) Separation of Polyphenols and Proteins from Flaxseed Hull Extracts by Coagulation and Ultrafiltration. Journal of Membrane Science 442, 177-186.

[11] Baldisserotto, A., Malisardi, G., Scalambra, E., Andreotti, E., Romagnoli, C., Vicentini, C.B., Manfredini, S. and Vertuani, S. (2012) Synthesis, Antioxidant and Antimicrobial Activity of a New Phloridzin Derivative for Dermo-Cosmetic Applications. Molecules 17(11), 13275-13289.

[12] Akram, S., Amir, R. M., Nadeem, M., Sattar, M. U., Faiz, F. (2012) Antioxidant potential of black tea (Camellia sinensis L.) e a review. Pakistan Journal of Food Science 22(3), 128-132.

[13] Tuberoso, C. I. G., Boban, M., Bifulco, E., Budimir, D., Pirisi, F. M. (2013) Antioxidant capacity and vasodilatory properties of Mediterranean food: the case of Cannonau wine, myrtle berries liqueur and strawberry-tree honey. Food Chemistry 140(4), 686-691.

[14] Yin, H., Xu, L., Porter, N. A. (2011) Free radical lipid peroxidation: mechanisms and analysis. Chemical Reviews 111(10), 5944-5972.

[15] Carvalho C. D., Costa, H. S., Albuquerque, G. T., Ramos, F., Castilho, M.C., SanchesSilva, A. (2015) Advances in phenolic compounds analysis of aromatic plants and their potential applications. Trends in Food Sc. \& Tech 45(2), 336-354.

[16] Boulekbache, M. L., Meudec, E., Mazauric, J. P., Madani, K., Cheynier, V. (2013) Qualitative and Semiquantitative Analysis of Phenolics in Eucalyptus globulus Leaves by Highperformance Liquid Chromatography Coupled with Diode Array Detection and massspectrometry. J. Phytochem. Anal 24(2), $162-170$.

[17] Desmond, J. T. (2017) Introduction to skin aging. Journal of Tissue Viability 26(1), 37-46.

[18] Bhoi, A. K., Sherpa, K. S., \& Khandelwal, B. (2018). Arrhythmia and ischemia classification and clustering using QRS-ST-T (QT) analysis of electrocardiogram. Cluster Computing, 21(1), 1033-1044.

[19] Reddy, A. V., Krishna, C. P., \& Mallick, P. K. (2019). An image classification framework exploring the capabilities of extreme learning machines and artificial bee colony. Neural Computing and Applications, 1-21.

[20] Bisoy, S. K., Mallick, P. K., \& Mishra, A. Fairness Analysis of TCP Variants in Asymmetric Network. International Journal of Engineering \& Technology, 7(2.12), 231-233. 
[21] Mallick, P. K., Mishra, D., Patnaik, S., \& Shaw, K. (2016). A semi-supervised rough set and random forest approach for pattern classification of gene expression data. International Journal of Reasoningbased Intelligent Systems, 8(3-4), 155-167.

[22] Mallick, P. K., Mohanty, B. P., \& Jha, S. A novel approach using. Supervised and Unsupervised Learning" to prevent the adequacy of Intrusion Detection Systems", International Journal of Engineering \& Technology, 7(3.34), 474-479.

[23] Satapathy, S. K., Mishra, S., Sundeep, R. S., Teja, U. S. R., Mallick, P. K., Shruti, M., \& Shravya, K. (2019). Deep learning based image recognition for vehicle number information. International Journal of Innovative Technology and Exploring Engineering, 8, 52-55.

[24] Mallick, P. K., Kar, S. K., Mohanty, M. N., \& Kumar, S. S. (2015). Use of histogram approach in color band detection for electrical passive component. International Journal of Applied Engineering Research, 10(44), 31446-31450.

[25] Mishra, S., Mallick, P. K., Tripathy, H. K., Bhoi, A. K., \& González-Briones, A. (2020). Performance Evaluation of a Proposed Machine Learning Model for Chronic Disease Datasets Using an Integrated Attribute Evaluator and an Improved Decision Tree Classifier. Applied Sciences, 10(22), 8137.

[26] Bhoi, A. K., Sherpa, K. S., \& Mallick, P. K. (2014, April). A comparative analysis of neuropathic and healthy EMG signal using PSD. In 2014 International Conference on Communication and Signal Processing (pp. 1375-1379). IEEE.

[27] Bhoi, A. K., Sherpa, K. S., Khandelwal, B., \& Mallick, P. K. (2019). T Wave Analysis: Potential Marker of Arrhythmia and Ischemia Detection-A Review. In Cognitive Informatics and Soft Computing (pp. 121130). Springer, Singapore.

[28] Mishra, S., Mallick, P. K., Jena, L., \& Chae, G. S. (2020). Optimization of Skewed Data Using SamplingBased Preprocessing Approach. Frontiers in Public Health, 8.

[29] Bhoi, A. K., \& Sherpa, K. S. (2016). Statistical analysis of QRS-complex to evaluate the QR versus RS interval alteration during ischemia. Journal of Medical Imaging and Health Informatics, 6(1), 210-214.

[30] Mishra, S., Tripathy, H. K., Mallick, P. K., Bhoi, A. K., \& Barsocchi, P. (2020). EAGA-MLP-An Enhanced and Adaptive Hybrid Classification Model for Diabetes Diagnosis. Sensors, 20(14), 4036.

[31] Bhoi, A. K., Mallick, P. K., Liu, C. M., \& Balas, V. E (Eds.) (2021). Bio-inspired Neurocomputing, Springer.

[32] Oniani, S., Marques, G., Barnovi, S., Pires, I. M., \& Bhoi, A. K. (2020). Artificial Intelligence for Internet of Things and Enhanced Medical Systems. In Bio-inspired Neurocomputing (pp. 43-59). Springer, Singapore.

[33] Marques, G., Bhoi, A.K., Albuquerque, V.H.C. de, K.S., H. (Eds.) (2021). IoT in Healthcare and Ambient Assisted Living, Springer

[34] Marques, G., Miranda, N., Kumar Bhoi, A., Garcia-Zapirain, B., Hamrioui, S., \& de la Torre Díez, I. (2020). Internet of Things and Enhanced Living Environments: Measuring and Mapping Air Quality Using Cyber-physical Systems and Mobile Computing Technologies. Sensors, 20(3), 720. 